\title{
IMAGING OF VENOUS THROMBOEMBOLIC DISEASE
}

\author{
L Ness, Consultant Radiologist \\ Royal Lancaster Infirmary
}

\section{INTRODUCTION}

There is a spectrum of venous thromboembolic disease ranging from deep vein thrombosis (DVT) to potentially fatal pulmonary embolism (PE). Lack of reliable clinical findings, coupled with anxiety to avoid missing these diagnoses, has resulted in increased referral rates for diagnostic imaging. Traditional $\mathrm{X}$-ray studies lack specificity and can be hazardous. A wealth of new research information is available to inform rational clinical management and new radiology techniques can provide more accurate diagnoses with less hazard in some cases. This article considers new imaging strategies and techniques.

\section{CLINICAL ASSESSMENT}

There is a widely-held belief that physical examination is completely unreliable in suspected DVT, but this is based upon studies from the $1960 \mathrm{~s}^{(1)}$. Recent studies highlight the importance of history and physical examination as predictors of lower extremity DVT and when combined with clinical risk factors, can guide appropriate use of imaging in those who need it most whilst excluding patients for whom it is not relevant ${ }^{2.33}$. Common clinical situations such as calf tenderness and chronic unilateral swelling (in the absence of risk factors), clinically evident cellulitis and lower limb joint pain were not associated with acute DVT in a large series ${ }^{(2)}$.

The use of the D-dimer blood test promises to be a useful screening tool due to its very high negative predictive value for thrombus. It has been predicted that inappropriate lower limb imaging referrals could be reduced by $35 \%$ if D-dimer levels are normal ${ }^{(4)}$.

The non-invasive nature of modern imaging techniques in suspected DVT and PE has lowered the threshold for referral, resulting in overuse and poor clinical justification. Using clinical protocols will enable cost-effective and resource- efficient investigation. Clinical assessment and D-dimer are covered in detail elsewhere in this issue.

\section{LOWER LIMB VENOUS IMAGING}

Virtually all pulmonary emboli arise from deep pelvic and lower limb veins. Spontaneous upper limb thrombus is far less common and rarely causes significant PE. Contrast venography was considered the gold standard investigation for detecting lower limb thrombus during previous decades mainly because there were no reliable alternatives, but it is not ideal (see table) and is now only reserved for occasional problem solving.

Ultrasound venography (USV) dates from the 1980s and has emerged as the preferred first line imaging test of suspected lower limb DVT, benefiting from equipment developments and improved operator technique and expertise. A combined colour/spectral doppler and compression technique is routinely employed throughout the $\mathrm{UK}^{(5)}$ and is the initial investigation of choice in FGH, WGH and RLI. Scanning commences at the iliac veins, continues along the femoropopliteal veins then, depending upon operator expertise, may continue into the paired calf veins although this can prolong the examination time. Lack of venous compressibility and visible intraluminal thrombus support a diagnosis of acute DVT. Some workers report heterogenous intraluminal echoes, multiflow channels and collateral vessel formation as evidence of chronic DVT but it can be difficult to demonstrate these $\operatorname{signs}^{(2)}$. A diagnostic study is nearly always achieved whereas contrast venography may not be technically possible in up to $20 \%$ of patients. Additionally ultrasound may reveal alternative causes of lower limb swelling ${ }^{(6)}$. Recent large series have found high sensitivity, specificity and positive predictive values approaching $100 \%$ for USV detection of thigh vein thrombosis in symptomatic patients. These values reduce for thrombus below knee level ${ }^{(7)}$.

\begin{tabular}{|c|c|}
\hline contrast venography & ultrasound venography \\
\hline non-specific findings in vessel occlusion & direct imaging of vessels reveals any contained thrombus \\
\hline underfilling of veins occurs for reasons other than thrombus & flow confirmed by colour or spectral doppler \\
\hline uncomfortable for patient & $\begin{array}{c}\text { well tolerated } \\
\text { nompletely safe } \\
\text { uses ionising radiation }\end{array}$ \\
\hline $\begin{array}{c}\text { risk of precipitating thrombus in 1-2\%. } \\
\text { Contrast reactions may occur }\end{array}$ & no risk of complications \\
\hline $\begin{array}{c}\text { only examines the venous system } \\
\text { usually shows distal calf veins but technical failure occurs in 20\% }\end{array}$ & $\begin{array}{c}\text { cross-sectional imaging will demonstrate alternative diagnoses in } \\
12 \% \text { of cases (ruptured Baker's cyst, haematoma or abscess) } \\
\text { examination of pelvic and thigh veins successful in } 100 \% . \\
\text { Visualisation of calf veins possible although time-consuming } \\
\text { and dependent on operator expertise }\end{array}$ \\
\hline
\end{tabular}


Perceived inferiority of USV in detecting below-knee thrombus caused initial reluctance to accept the technique, but the wider debate over the precise significance of isolated calf thrombus persists. The prevalence of DVT in symptomatic patients in one study was $15 \%$ of which $85 \%$ was located above knee level. Thus isolated calf thrombus is uncommon and it would prolong examination time to try to find it in a relatively small number of patients ${ }^{(8)}$. Emboli from calf thrombus would cause small PE which are non-fatal and probably not clinically significant ${ }^{(9)}$. The risk of PE following negative thigh USV examination is extremely low $(0.3 \%)$ in symptomatic patients ${ }^{(8)}$. It is known that undetected calf thrombus can propagate into proximal veins in a minority of cases $(7.7 \%)$ so it has been proposed that selected patients remaining symptomatic after initial USV should have a repeat study at approximately 10 days ${ }^{(10)}$. A recent survey of 65 UK radiology departments revealed that only two routinely perform such follow-up scans ${ }^{(11)}$.

\section{PULMONARY CIRCULATION IMAGING}

The traditional radiological diagnosis of PE was dependant upon chest $\mathrm{x}$-ray (CXR), ventilation/perfusion scan (V/Q) and catheter pulmonary angiography.

CXR The chest film is a non-specific test in acute PE and its main role is to exclude other disease. However it has been shown that a normal CXR can predict a diagnostically useful V/Q result ${ }^{(12)}$.

V/Q scans Combined V/Q scanning is a non-invasive investigation available to most hospitals in the UK and is well tolerated. Scan results are categorised into high, intermediate and low probabilities according to agreed criteria. If a V/Q scan is read as high probability and there is also high clinical suspicion then the certainty that PE is present exceeds $96 \%$. Similarly if the study is read as low probability or normal there is a greater than $98 \%$ chance that the patient does not have PE. Unfortunately in reality $70 \%$ of V/Q scans are classed as indeterminate due to co-existing cardiopulmonary disease and furthermore they have a reported $30 \%$ interobserver variation. The only prospect of obtaining a definitive diagnosis in the past was to proceed to catheter pulmonary angiography ${ }^{(13)}$.
Catheter pulmonary angiography This has been the gold standard test for evaluating suspected PE but is only available in $15 \%$ of UK hospitals. It is invasive, potentially hazardous and subject to interobserver disagreement in reporting. A recent survey of UK practice found that most patients were managed according to V/Q results alone and few underwent pulmonary angiography ${ }^{(14)}$

CT pulmonary angiography (ctpa) When spiral CT scanners were introduced in the early 1990s it became possible to perform CT angiography of the pulmonary circulation. This type of scanner acquires volume data rather individual slices and can rapidly image a large volume of tissue during transit of intravenous contrast medium. The latest development of spiral CT is multidetector CT where several detectors sample the same $\mathrm{x}$-ray beam simultaneously providing even faster acquisition and exquisite tissue detail. Multidetector scanners will be installed in RLI (2001) and FGH (2002). CTPA is non-invasive and is progressively replacing catheter pulmonary angiography.

a) technique CTPA requires optimum contrast opacification of the pulmonary circulation to outline any contained emboli. Intravenous contrast injected into a peripheral vein has a variable transit time which can be prolonged in congestive cardiac failure, but modern scanners can detect contrast arrival and commence scanning at the correct moment. Even with rapid scan times it is important to avoid any voluntary movement and breath holding is required for 10 seconds minimum (ideally 20 seconds) after preliminary hyperventilation. Scanning is performed during maximal inspiration when compressed alveolar vessels cause increased pulmonary arterial resistance. This maximizes vessel opacification. Scanning commences at the level of the aortic arch and extends below the inferior pulmonary veins.

Some workers in North America have extended this technique to include indirect CT venography of IVC, pelvic and thigh veins using the contrast already in the circulation from the initial CTPA. It is known that a small proportion of patients $(8 \%)$ suspected of having PE but negative CTPA will have co-existing lower limb DVT and this modification could be appropriate in selected cases where poor cardiopulmonary reserve might be critically compromised in the event of embolism ${ }^{(9.15}$

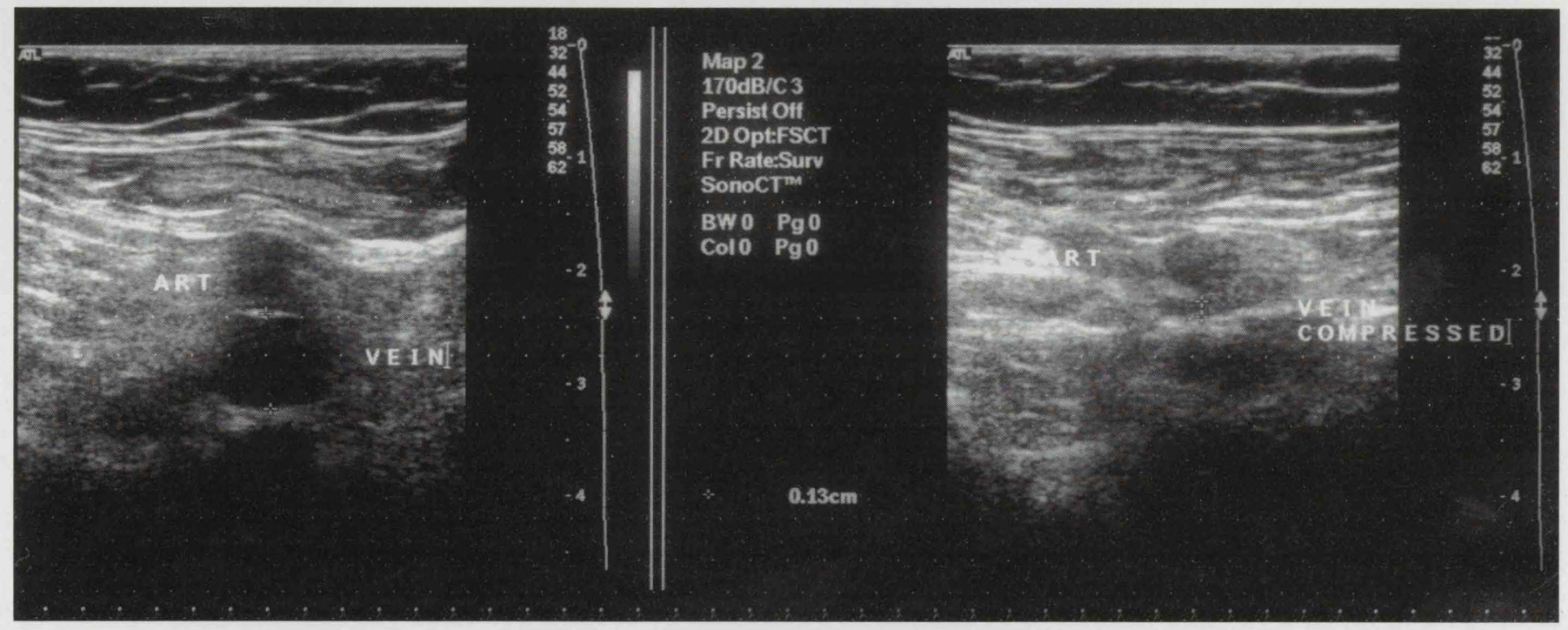

Figure 1 Illustration of the compression ultrasound technique showing a deep thigh vein before (left) and after (right) compression 


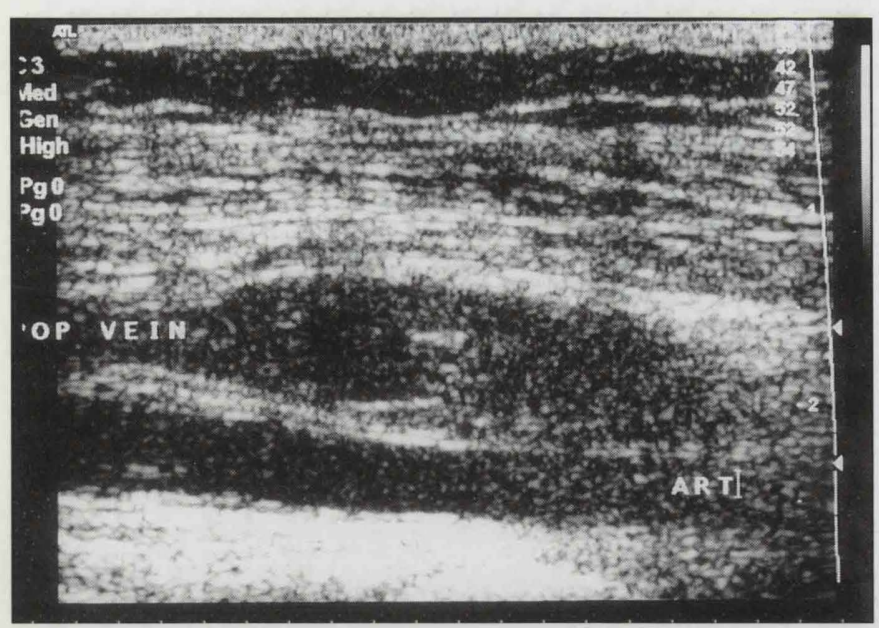

Figure 2 Direct ultrasound visualisation of thrombus distending a popliteal vein with the adjacent artery (narrower vessel) lying below

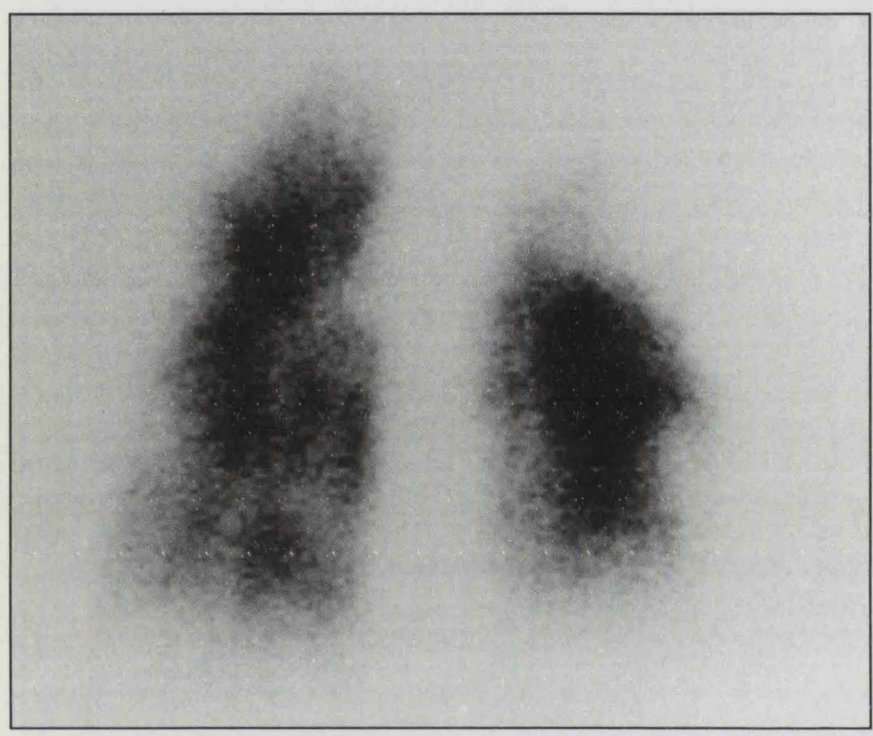

Figure 3 A nuclear medicine lung perfusion scan showing widespread perfusion defects typical of multiple pulmonary emboli

b) results CTPA can achieve opacification of vessels down to 2-3 mm diameter with good to excellent technical quality in $95 \%$ of patients ${ }^{(9)}$. A suboptimal examination rate of $2-4 \%$ is similar to that of catheter pulmonary angiography and the commonest cause is severe dyspnoea but this is offset by a lower percentage of non-diagnostic CTPA as alternative diagnoses are suggested by direct evaluation of chest structures in up to $33 \%$ of cases $^{(16)}$. Sensitivity of CTPA for PE (75-92\%) is far superior to V/Q scans and interobserver agreement is also better amongst $\mathrm{CT}$ readers ${ }^{(16)}$

c) indications A safer, faster, more convenient noninvasive replacement for conventional pulmonary angiography combined with a higher diagnostic yield will be welcomed by clinicians faced with the difficulties of diagnosing PE. This attraction must however be tempered by reality. CTPA will compete with an ever increasing CT workload and is not an unlimited resource. Furthermore CT is responsible for a significant proportion of the medical radiation burden received by the population and CTPA specifically results in a high dose to the radiosensitive female breast. V/Q scanning should continue as the initial investigation for $\mathrm{PE}$ in many patients as a normal or high probability perfusion scan result has considerable value in management leaving CTPA as a

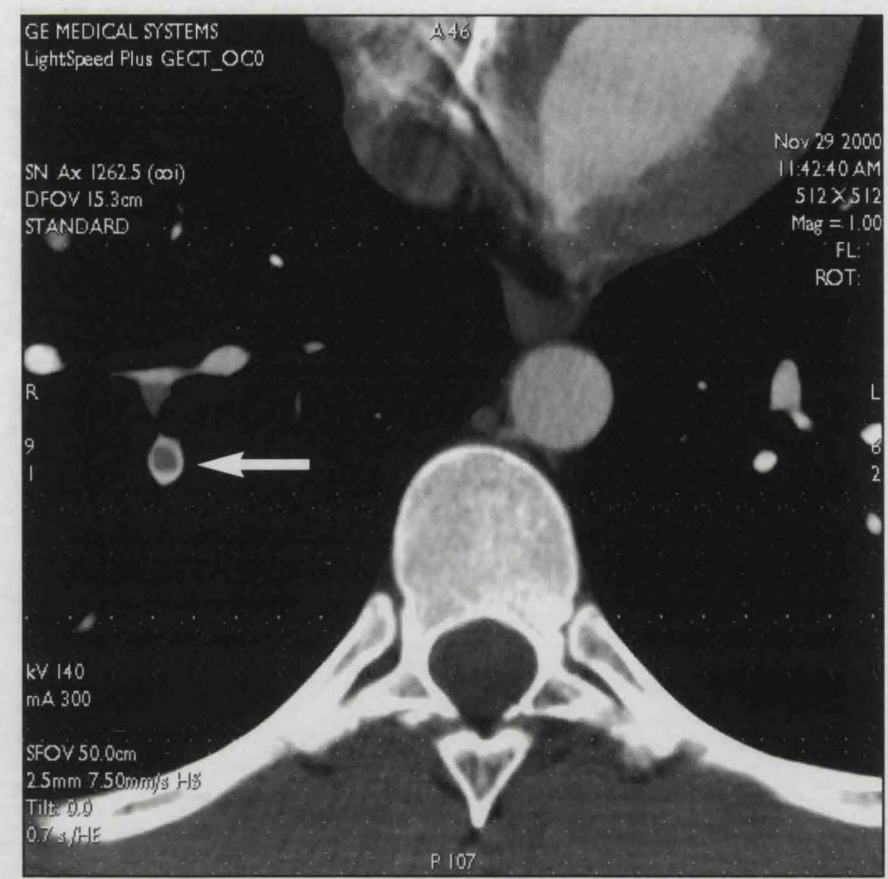

Figure 4 An axial slice from a CTPA showing thrombus within a medium-sized pulmonary artery

(Courtery of GE Medical Systems)

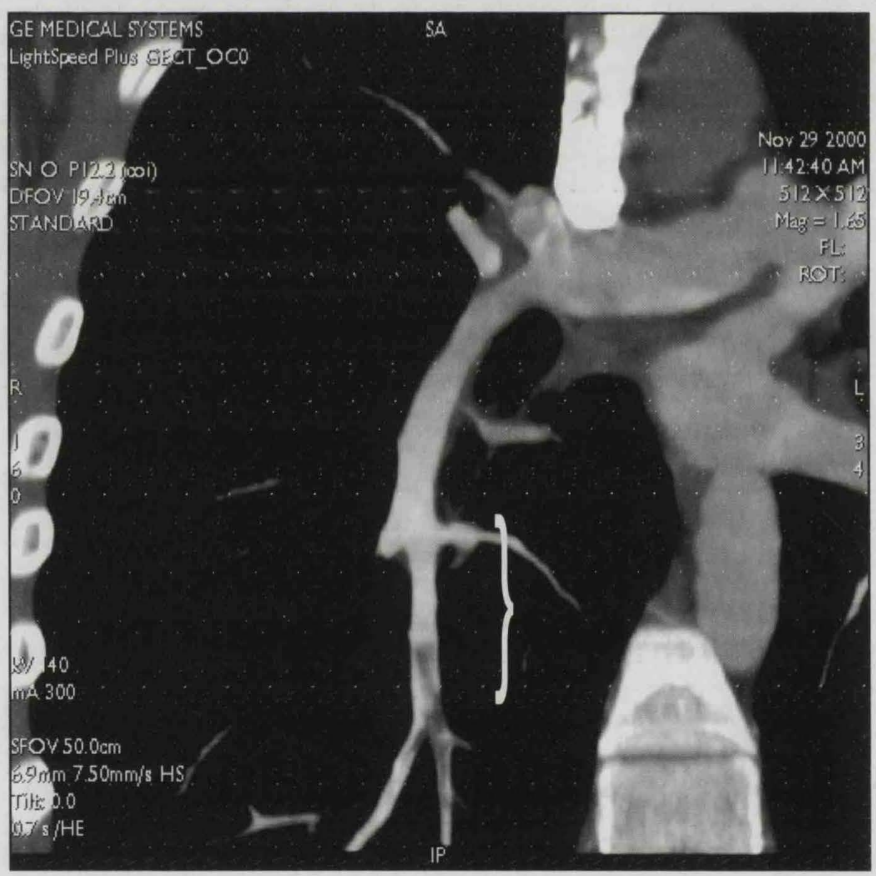

Figure 5 A coronal CTPA reconstruction showing emboli within an inferior pulmonary artery extending into a branch vessel

(Courtesy of GE Medical Systems)

second line test for indeterminate V/Q and emergency situations.

\section{FUTURE DEVELOPMENTS}

Modern imaging is already making the diagnosis of PE and DVT more accurate and convenient. Advances in ultrasound design and technique will doubtless improve the visualisation of thrombus. CTPA is already having a major impact in the diagnosis of PE although the use of ionising radiation is undesireable and magnetic resonance pulmonary angiography is now advancing from a research tool to a clinically relevant test ${ }^{(17)}$. MR contrast agents do not have the safety risks 
associated with radiological contrast media and so if faster MR scanners with improved spatial resolution can be developed then the ideal imaging test would be possible. Ultrafast 3D MR angiography using blood pool imaging would enable a one-stop totally non-invasive approach to visualise lower extremity veins and pulmonary circulation.

Perhaps the greatest future prospect is that these noninvasive procedures would give clearer understanding of the entire enigma of venous thromboembolic disease. It is known that many patients with autopsy proven PE never had the diagnosis considered during life and conversely $63 \%$ of patients diagnosed as having the disease did not have it at autopsy ${ }^{(18)}$. Winston et $a l^{(19)}$ reviewed 1879 consecutive contrast enhanced CT scans and found incidental PE in nearly $1 \%$ of the population. We may soon be able to study venous thrombosis in greater detail than ever before and determine its true significance in a given individual so that our energies can be focussed on those who are at risk from its most extreme outcome.

\section{REFERENCES}

1 McLachlin JA, Richards T, Paterson JC. An evaluation of clinical signs in the diagnosis of venous thrombosis. Arch Surg 1962;85:738-44

2 Glover JL, Bendick PJ. Appropriate indications for venous duplex ultrasonographic examinations. Surgery 1996;120(4):725-30

3 Criado E, Burnham CB. Predictive value of clinical criteria for the diagnosis of deep vein thrombosis. Surgery $1997 ; 122(3): 578-83$

4 Bradley M, Bladon J, Barker H. D-dimer assay for deep vein thrombosis: its role with colour doppler sonography. Clin Radiol 2000;55(7):525-7

5 Donnelly R, Hinwood D, London NJM. ABC of arterial and venous disease: non-invasive methods of arterial and venous assessment. Br Med J 2000;320:698-701

6 Banerjee A. The assessment of acute calf pain. Postgrad Med J 1997;73(856):86-8

7 Cornuz J, et al. Deep venous thrombosis: complete lower extremity venous US evaluation in patients without known risk factors - outcome study. Radiology 1999;211(3):637-41
8 Gottlieb RH, et al. Clinical outcomes of untreated symptomatic patients with negative findings on sonography of the thigh for deep vein thrombosis: our experience and a review of the literature. AJR 1999;172(6):1601-4

9 Cham MD, Yankelevitz DF et al. Deep venous thrombosis: detection by using indirect CT venography. The Pulmonary Angiography/Indirect CT Venography Cooperative Group. Radiology 2000;216(3):744-51

10 Masuda EM, Kessler DM, Kistner RL et al. The natural history of calf vein thrombosis: lysis of thrombi and development of reflux. J Vasc Surg 1998;28(1):67-73

11 Walsh PF, Lauder JC. The radiological investigation of suspected lower limb deep vein thrombosis. Clin Radiol 2000;55:79(letter)

12 Forbes KPN, Reid JH, Murchison JT. Do preliminary chest $\mathrm{x}$-ray findings define the optimum role of pulmonary scintigraphy in suspected pulmonary embolism? Clin Radiol 2001;56(5)397-400

13 PIOPED Investigators. Value of the ventilation/perfusion scan in acute pulmonary embolism. JAMA 1990;263:2753-9

14 Burkill GJ, Bell JR, Padley SP. Survey on the use of pulmonary scintigraphy, spiral $\mathrm{CT}$ and conventional pulmonary angiography for suspected pulmonary embolism in the British Isles. Clin Radiol 1999;54(12):807-10

15 Loud PA, Katz DS et al. Combined CT venography and pulmonary angiography in suspected thromboembolic disease: diagnostic accuracy for deep venous evaluation. AJR 2000;174(1):61-5

16 Remy-Jardin M, Remy J. Spiral CT angiography of the pulmonary circulation. Radiology 1999;212(3):615-36

17 Hoffman U, Schima W, Herold C. Pulmonary magnetic resonance angiography. Eur Radiol 1999;9(9):1745-54

18 Stein PD, Henry JW. Prevalence of acute pulmonary embolism among patients in a general hospital and at autopsy. Chest 1995;108:978-81

19 Winston CB et al. Incidental pulmonary emboli detected at helical CT: effect on patient care. Radiology 1996;201:2327 